\title{
A FORMAÇÃO DA PERCEPÇÃO DE RESPONSABILIDADE SOCIAL CORPORATIVA EM CLIENTES BANCÁRIOS
}

\section{THE FORMATION OF THE PERCEPTION OF CORPORATE SOCIAL RESPONSIBILITY BY BANKING CLIENTS}

\footnotetext{
Tiago Zardin Patias

Professor Adjunto da Universidade Federal do Pampa - UNIPAMPA

Santana do Livramento, RS, Brasil

E-mail: tiagopatias@unipampa.edu.br

Marta Von Ende

Professora do Colégio Politécnico da Universidade Federal de Santa Maria - UFSM

Santa Maria, RS, Brasil

E-mail: alinecrespo@uscs.edu.br

Clandia Maffini Gomes

Professora e Coordenadora do PPGA - Universidade Federal de Santa Maria - UFSM

Santa Maria, RS, Brasil

E-mail: clandia@smail.ufsm.br

Lúcia Rejane da Rosa Gama Madruga

Professora do PPGA - Universidade Federal de Santa Maria - UFSM

Santa Maria, RS, Brasil

E-mail: luciagm@ufsm.br
}

\section{RESUMO}

Este artigo objetiva compreender como clientes do setor bancário formam suas percepções de Responsabilidade Social Corporativa (RSC). Testouse um modelo causal numa amostra de 242 clientes bancários, para explicar como os consumidores em geral formam suas percepções sobre a RSC, incluindo três variáveis como antecedentes: a congruência entidade-RSC, a atribuição de motivações corporativas e a credibilidade da entidade. Essas variáveis são consideradas condicionantes diretos da imagem da RSC das empresas. Confirmouse a implicação da congruência RSC-organização nas variáveis moderadoras atribuição motivacional e credibilidade corporativa, bem como diretamente na percepção da RSC. A influência da atribuição motivacional na credibilidade corporativa foi confirmada, no entanto não foi confirmado seu efeito direto na percepção da RSC. Por fim, foi também confirmado o efeito da credibilidade corporativa na percepção da RSC.

Palavras-chave: Responsabilidade social corporativa. Imagem. Bancos.

\section{ABSTRACT}

This article aims to understand how banking customers form their perceptions of corporate social responsibility (CSR). We tested a causal model in a sample of 242 bank clients to explain how mainstream consumers form their perceptions of CSR, including three variables as antecedents: the entity-CSR congruence, the allocation of corporate motives and credibility of the organization. These variables are considered direct constraints of the CSR image of companies. Confirmed the involvement of the RSCorganization congruence in motivational assignment moderating variables and corporate credibility, as well as directly in the perception of CSR. The influence of motivational assignment in corporate credibility has been confirmed, however was not confirmed their direct effect on the perception of CSR. Finally, it was also confirmed the effect of corporate credibility in the perception of CSR.

Keywords: Corporate social responsibility. Image. Banks.

Data de submissão: 29 de abril de 2016.

Data de aprovação: 20 de maio de 2017. 


\section{INTRODUÇÃO}

As empresas estão enfrentando um novo paradigma de negócios e de marketing, no qual a importância da reputação de seus produtos no mercado dá lugar para uma série de novas demandas sociais, que ressaltam o valor dos atributos empresariais a nível corporativo (HE; BALMER, 2007).

Currás (2010) aponta que, nesse contexto, os atributos organizacionais e de marca são mais duradouros e resistentes às pressões competitivas do que os atributos próprios do produto ou serviço, complementando que, como consequência dessa tendência, tem se produzido em âmbito acadêmico um forte desenvolvimento da investigação em termos de conceitos corporativos estreitamente vinculados a empresa em seu conjunto, como a identidade, a imagem, a reputação, a comunicação ou a marca corporativas, entre outros.

É dentro desta perspectiva que a ideia da Responsabilidade Social Corporativa (RSC) vem consolidando-se não apenas no meio acadêmico, mas também empresarial. Nasceu sob certo ceticismo, mas avançou, na medida em que a sociedade clama por igualdade, justiça e melhores condições de vida para viver com dignidade. Carson, em 1962, foi quem primeiramente fez uma alerta dos efeitos industriais, principalmente de produtos químicos, sobre a humanidade (MARCO; HOLLINGWORTH; DURHAM, 1987). Esse movimento expandiu-se ao longo do tempo, exigindo das empresas ações efetivas em prol da preservação dos recursos naturais.

A RSC foi primeiramente definida por Howard R. Bowen, em 1953, como aquela que "se refere às obrigações dos empresários de buscar determinadas políticas, de tomar certas decisões ou de seguir linhas de ação desejáveis em termos de objetivos e valores para a nossa sociedade" (CARROLL, 1999, p. 270).

Apesar de não haver um consenso na definição do termo, tendo em vista que ao longo do tempo vem se aperfeiçoando e incorporando novos elementos (DAHLSRUD, 2008), o conceito de Carroll (1979, p. 500) tem sido amplamente utilizado e aceito até os dias de hoje, onde afirma que "responsabilidade social dos negócios envolve as expectativas da sociedade em relação às organizações, nos aspectos econômico, legal, ético e discricionário [filantrópico], em dado momento no tempo".

Ashley et al. (2003), mais recentemente, definem a RSC em termos do compromisso que uma organização deve ter para com a sociedade, expresso por meio de atos e atitudes que a afetem positivamente, agindo de forma proativa e coerentemente no que tange a seu papel específico na sociedade e a sua prestação de contas para com ela.

Cabe destacar que a pressão da sociedade, do consumidor e do governo deu um impulso inicial para que as organizações empresariais incluíssem a sustentabilidade e as questões ambientais em seus critérios de tomada de decisão e estratégias (SERPA; FOURNEAU, 2007; SANDHU, 2010; BONN; FISHER, 2011). $\mathrm{Na}$ década de 1990, movimentos de consumidores favorecem produtos amigos do ambiente, com manifestações de vontade de incorporar critérios ambientais nas decisões de compra e dispostos a pagar mais por produtos mais ecológicos.

Delios (2010) complementa, apontando que as organizações empresariais, enquanto entidades sociais podem e devem arriscar-se a ter preocupações sociais, não só por uma questão de altruísmo ou porque defendem valores morais e éticos, mas porque a preocupação com os colaboradores e a comunidade cria oportunidades para alcançar vantagens competitivas.

Atualmente inúmeras ações são desenvolvidas pelas organizações para minimizar seus efeitos sobre a sociedade, no entanto, nem todas as ações são percebidas. Estudos têm sido desenvolvidos no nível dos 
stakeholders para entendê-los e saber as consequências (SEN; BHATTACHARYA, 2001; IDOWU; PAPASOLOMOU, 2007; PEDERSEN; NEERGAARD, 2009; LYRA; SOUZA, 2013).

No universo de empresas que desenvolvem intervenções e promovem a responsabilidade social, os bancos podem ser destacados por algumas razões específicas do setor. As características do setor bancário, aliadas a seu baixo potencial de impacto ambiental, permite que tenham mais flexibilidade para a realização de ação social com mais visibilidade comparativamente a outros setores (CRISÓSTOMO; FREIRE; SOARES, 2012).

Conforme apontam Guimarães Filho e Gomes (2010), o setor bancário, por seu poder econômico, sua capilaridade, tecnologia operacional e de gestão, pode ser forte indutor na disseminação da prática eficaz da RSC.

Pesquisando bancos espanhóis, Bravo, Matute e Pina (2012) verificaram que tais empresas divulgam RSC com o objetivo de construir identidade associada à RSC e obter legitimação. No Brasil, Crisóstomo, Freire e Vasconcellos (2011) encontraram resultados que indicam o setor bancário com a mais elevada RSC no país.

Nesse sentido, considerando a importância dessa nova realidade social, tanto do ponto de vista acadêmico quanto empresarial, torna-se relevante compreender o papel da responsabilidade social corporativa na sociedade atual e de que forma os clientes percebem e integram a RSC na sua tomada de decisões. Na direção desse entendimento, este artigo tem por objetivo compreender como clientes do setor bancário geralmente formam suas percepções de RSC.

O artigo está estruturado em cinco seções, incluindo esta introdução. Na seção 2 são apresentadas algumas definições e modelos conceituais relacionados à formação da imagem de responsabilidade social corporativa. A seção 3 destina-se a apresentar os aspectos metodológicos do presente estudo. A apresentação e discussão dos resultados são realizadas na seção 4. Por fim, na seção 5 são apresentadas as considerações finais do estudo.

\section{REFERENCIAL TEÓRICO}

\section{Responsabilidade Social Corporativa (RSC)}

No atual cenário de competitividade, onde a questão da sustentabilidade tem sido cada vez mais debatida no meio acadêmico e empresarial, as organizações se deparam com uma pressão cada vez maior para tomar iniciativas e reconhecer suas responsabilidades na área social. Dunphy (2003) destaca que uma questão que tem emergido é se a adoção pelas empresas de estratégias de responsabilidade social, que apoiam o desenvolvimento sustentável sob o ponto de vista econômico, social e ambiental, pode desempenhar um papel fundamental na geração de vantagem competitiva e melhor desempenho.

A definição de desenvolvimento sustentável existe desde a década de 70 , mas, segundo Morimoto, Ash e Hope (2005), tornou-se mais conhecida a partir de 1987, quando a Comissão Mundial de Meio Ambiente e Desenvolvimento publicou o Relatório Brundtland. Esse relatório teve como foco alertar para a urgência de não se esgotar os recursos naturais e degradar o meio ambiente. Assim, o conceito de desenvolvimento sustentável mais difundido é o da Comissão Brundtland, que considera que o desenvolvimento sustentável é 
aquele que satisfaz as necessidades do presente sem comprometer a capacidade das gerações futuras de satisfazerem suas próprias necessidades (WCED, 1987).

Sob essa perspectiva, as empresas que até então tinham como paradigma o foco no lucro (geração de valor para os acionistas), com o conceito de desenvolvimento sustentável as corporações passam a incorporar ao seu objetivo os componentes: proteção ao meio-ambiente e igualdade social. Ao conjunto dessas três dimensões é chamado Triple Bottom Line, também conhecido como Tripé da sustentabilidade ou People Planet Profit (LEMME, 2005).

Apesar da recente disseminação do discurso da sustentabilidade, Hart e Milstein (2004) apontam que grande parte dos executivos ainda considera o desenvolvimento sustentável uma espécie de carga necessária, uma vez que envolve regulação, custos e responsabilidades onerosas. Os autores destacam, ainda, que a partir de argumentos legais ou morais para a ação das empresas, os executivos inevitavelmente subestimam as oportunidades estratégicas de negócios associados a este importante tema.

No contexto empresarial, desenvolvimento sustentável tem sido denominado também como responsabilidade corporativa e sustentabilidade corporativa. Para Hedstrom et al. (1999), sustentabilidade corporativa é uma abordagem capaz de criar prosperidade com horizontes de longo prazo, através da integração de estratégias voltadas para o crescimento econômico mundial, a qualidade ambiental voltada para a preservação do ecossistema e ao aumento da capacidade econômica da população mundial em busca da qualidade de vida.

Apesar da crescente percepção de que as empresas devem tratar a questão da sustentabilidade, não há uma definição clara na literatura sobre o significado de sustentabilidade no contexto organizacional, de forma que uma série de definições tem sido apresentada.

Dyllick e Hockerts (2002) definem sustentabilidade corporativa como atender as necessidades das partes interessadas diretas e indiretas da empresa (tais como, acionistas, funcionários, clientes, grupos de pressão, comunidades, etc), sem comprometer a sua capacidade de atender também as necessidades futuras das partes interessadas.

Van Marrewijk (2003) acredita ser importante reconhecer as diferenças entre os termos sustentabilidade empresarial e responsabilidade social corporativa (RSC), mesmo admitindo que muitos autores os considerem sinônimos. No passado, o termo sustentabilidade estava ligado a aspectos ambientais apenas, enquanto a responsabilidade social referia-se a questões sociais. Segundo o autor, a sustentabilidade teria o foco sobre conflitos de interesse e estaria relacionada à criação de valor, a gestão ambiental e do capital humano, do sistema de produção limpo, por exemplo. Já a RSC se concentraria sobre o relacionamento entre pessoas e organizações, incluindo aspectos como transparência, diálogo com grupos de interesse e a apresentação dos resultados em sustentabilidade.

Existe uma variedade de definições sobre a RSC, apesar disso não há consenso em torno de um conceito amplamente aceito. Em que pese às inúmeras divergências, Carroll (1979) sugere que a RSC pode ser entendida como a expansão do papel empresarial além de seu escopo econômico e de suas obrigações legais.

A definição moderna para RSC, segundo Carroll (1999), data de 1953 com a publicação do livro "Responsabilidade Social dos Homens de Negócios", escrito por Howard R. Bowen. Em seu livro, Bowen (apud CARROLL, 1999, p. 270) questiona quais responsabilidades para com a sociedade se espera dos 
homens de negócios. O autor definiu responsabilidade social como a "obrigação dos homens de negócios de adotar diretrizes, decisões e linhas de ações desejáveis no âmbito dos objetivos e valores de nossa sociedade".

\section{A Formação de Imagem de RSC}

Matos (2005) afirma que já não é possível avaliar as empresas com os padrões tangíveis de ontem, pois referenciais intangíveis como marca, imagem, prestígio e confiabilidade decidem a preferência e garantem a continuidade.

Pérez e Rodríguez del Bosque (2013) apontam que a investigação acadêmica sobre a imagem da RSC tem centrando-se em conhecer a contribuição deste conceito aos resultados das empresas e, no que se refere a área de marketing, ao estudo da sua repercussão no comportamento do consumidor. Salientam ainda, que poucos trabalhos têm buscado conhecer o processo de formação da imagem de RSC dos clientes, o que limita o conhecimento existente sobre este conceito e dificulta a geração de estratégias eficazes para a gestão das empresas.

Rifon et al. (2004) propõem um modelo causal para entender como os consumidores em geral formam suas percepções sobre a RSC. Segundo os autores, o processo de formação da RSC inclui três variáveis, quais sejam: a congruência entidade-RSC, a atribuição de motivações corporativas e a credibilidade da entidade. Essas variáveis são consideradas condicionantes diretos da imagem da RSC das empresas.

No que se refere à congruência entidade-RSC, Pérez e Rodríguez del Bosque (2013) destacam que é comum para as organizações associarem seus produtos e marcas com outros objetos externos que acreditam que sejam positivamente avaliados pelos consumidores. Nan e Heo (2007) salientam que o objetivo dessa estratégia é causar uma transferência de imagem positiva de um atributo externo para a organização.

Essa associação entre a empresa e objetos ou atividades com uma avaliação positiva por parte dos indivíduos pode desencadear um processo de transferência de significado que, no campo específico da RSC, justifica porque o investimento em RSC tornou-se um movimento estratégico fundamental para as empresas.

Pérez e Rodríguez del Bosque (2013) destacam que a transferência não se produz de maneira direta nem igual em todas as situações, de forma que existem circunstâncias que mediam a intensidade da resposta positiva do cliente a vinculação da empresa com ações de RSC. Nessa linha, o modelo de Rifon et al. (2004) se apoia na teoria da atribuição (KELLEY, 1973) para explicar o efeito da congruência entre a empresa e a causa social na atitude do cliente para com a entidade. A teoria da atribuição postula que os consumidores cognitivamente inferem um motivo para o comportamento de patrocínio, assumindo que indivíduos são perceptores sociais que fazem inferências causais sobre os eventos que observam e experienciam.

Segundo Heider (1958), há dois tipos de fatores que podem moldar uma atribuição de motivo: (1) fatores pessoais internos ao ator (motivos intrínsecos) e (2) fatores situacionais externos ao ator (motivos extrínsecos).

Dessa forma, Rifon et al. (2004) apontam que, paralelas às motivações dos atributos externos como forma de transferência de significado, as corporações podem ser vistas como patrocinadoras de uma causa porque a empresa vê a causa tão digna, o que seria um motivo intrínseco, ou por causa de motivos extrínsecos, tais como aumento de lucro ou reputação. Dessa forma, as atribuições motivacionais feitas pelos 
clientes, ou seja, como os clientes percebem as motivações da organização para suas ações sociais, é um aspecto importante na compreensão da formação da percepção da RSC. Os autores salientam que as corporações normalmente querem evitar a percepção dos consumidores de motivação extrínseca, para evitar julgamentos sobre a exploração da empresa e outros resultados negativos.

A credibilidade da entidade nas suas ações de patrocínio também são contempladas no modelo de Rifon et al. (2004). Se os clientes perceberem que a motivação é menos altruísta e mais orientada a resultados e lucros, eles podem perceber a organização como menos crível. Park, Lee e Kim (2014) destacam que para ganhar a confiança dos consumidores, a empresa precisa cumprir suas responsabilidades legais e éticas.

Observa-se que há um relação de interdependência entre as variáveis constituintes do modelo de Rifon et al. (2004). O Quadro 1 apresenta as hipóteses do modelo de Rifon et al. (2004), bem como algumas referências de autores que sugerem a relação dos construtos para cada uma das hipóteses apresentadas.

Em linhas gerais, o modelo trabalha com seis hipóteses, quais sejam:

$H_{1}$ : A congruência simbólica entidade-RSC influi direta e positivamente nas atribuições motivacionais no desenvolvimento de sua responsabilidade social corporativa.

$H_{2}$ : A congruência simbólica entre a empresa e sua responsabilidade social corporativa influi direta e positivamente na credibilidade corporativa.

$H_{3}$ : A atribuição motivacional no desenvolvimento de sua responsabilidade social corporativa influi direta e positivamente na credibilidade corporativa.

$H_{4}$ : A congruência simbólica entidade-RSC influi direta e positivamente na percepção de responsabilidade social corporativa da empresa.

$H_{5}$ : A atribuição motivacional no desenvolvimento de sua responsabilidade social corporativa influi direta e positivamente na percepção de responsabilidade social corporativa da empresa.

$H_{6}:$ A credibilidade influi direta e positivamente, na imagem de responsabilidade social corporativa da empresa.

Quadro 1 - Hipóteses de relacionamento entre os construtos

\begin{tabular}{|l|l|}
\hline \multicolumn{1}{|c|}{ Relacionamento causal } & \multicolumn{1}{c|}{ Referências } \\
\hline Congruência $\rightarrow$ Atribuição & $\begin{array}{l}\text { Rifon et al. (2004); Bigné, Currás e Sánchez (2009); Öberseder, Schlegelmilch } \\
\text { e Murphy (2013); Peréz e Rodríguez del Bosque (2013) }\end{array}$ \\
Congruência $\rightarrow$ Credibilidade & $\begin{array}{l}\text { Rifon et al. (2004); Bigné, Currás e Sánchez (2009); Öberseder, Schlegelmilch } \\
\text { e Murphy (2013); Peréz e Rodríguez del Bosque (2013) } \\
\text { Rifon et al. (2004); Bigné, Currás e Sánchez (2009); Öberseder, Schlegelmilch } \\
\text { e Murphy (2013); Peréz e Rodríguez del Bosque (2013) }\end{array}$ \\
Congruência $\rightarrow$ Percepções RSC $\rightarrow$ Credibilidade & $\begin{array}{l}\text { Öberseder, Schlegelmilch e Murphy (2013); Peréz e Rodríguez del Bosque } \\
\text { (2013) }\end{array}$ \\
Atribuição $\rightarrow$ Percepções RSC & $\begin{array}{l}\text { Öberseder, Schlegelmilch e Murphy (2013); Peréz e Rodríguez del Bosque } \\
\text { (2013) }\end{array}$ \\
Credibilidade $\rightarrow$ Percepções RSC & $\begin{array}{l}\text { Bigné, Currás e Sánchez (2009); Öberseder, Schlegelmilch e Murphy (2013); } \\
\text { Peréz e Rodríguez del Bosque (2013); }\end{array}$ \\
\hline
\end{tabular}

Nota: As setas indicam uma influência positiva do construto anterior a seta sobre o construto após a seta.

Fonte: elaborado pelos autores. 


\section{MÉTODO}

A presente investigação caracteriza-se como descritiva, pois define, classifica e categoriza o fenômeno ou a amostra, determinando a relação entre duas ou mais variáveis ou eventos, ou o efeito de uma variável em outra, proporcionando informações sobre o componente médio de um grupo (MARCZYK; DEMATTEIO; FESTINGER, 2005).

Os participantes do estudo foram estudantes de instituições de ensino localizadas em Santa Maria RS, detentores de conta bancária, num total de 242 entrevistados, sendo que a amostra foi escolhida por conveniência. $O$ instrumento de coleta de dados utilizado neste estudo consiste em uma adaptação do questionário utilizado no estudo de Pérez e Rodríguez del Bosque (2013), que analisa a influência de características pessoais dos consumidores no modelo causal de Rifon et al. (2004).

A escala passou por um processo de tradução e tradução reversa, que significa que a escala foi traduzida do inglês para o português e depois do português para o inglês, e a tradução foi refeita até que não houvessem divergências entre o conteúdo das duas escalas. Assim, o questionário continha 4 construtos, todos baseados em pesquisa realizada por Pérez e Rodríguez del Bosque (2013) que utilizavam uma escala tipo Likert de 7 pontos, no qual o entrevistado deveria se posicionar entre $1=$ discordo totalmente até $7=$ concordo totalmente. O construto percepção da RSC foi medido utilizando uma escala composta de 22 itens reunidos em cinco dimensões - clientes, acionistas e conselhos, funcionários, sociedade e uma dimensão geral sobre questões legais e éticas que incluem responsabilidades corporativas. Os construtos, congruência empresa-CSR, atribuição motivacional e credibilidade corporativa foram medidos por meio de uma escala de 4 itens cada um deles. Além destes construtos, o questionário também apresentava algumas perguntas de caráter sócio demográfico, para a caracterização da amostra.

A coleta de dados ocorreu no mês de novembro de 2013 e posteriormente foram analisados com o apoio do software estatístico comercial SPSS, versão 21, para a produção de: i) estatísticas descritivas; ii) análise de consistência interna (Alfa de Cronbach). Todas as análises envolveram um nível de significância de $5 \%$.

Finalmente, foi utilizado o software IBM SPSS AMOS versão 21 para o emprego da Modelagem das Equações Estruturais (MEE), que é uma metodologia estatística multivariada para teste de hipótese de teoria sobre um fenômeno, incluindo as inter-relações entre os construtos. Por intermédio dessa metodologia, modelos podem ser testados estatisticamente de forma simultânea para verificar até que ponto o modelo teórico é consistente com os dados. Se houver ajuste adequado do modelo, utilizando uma série de critérios, pode-se considerar a plausibilidade das relações entre as variáveis, caso contrário, rejeita-se a hipótese de relação entre as variáveis estudadas (HAIR et al., 2009).

$\mathrm{Na}$ análise fatorial confirmatória (AFC), o ajuste do modelo foi identificado através dos seguintes índices e padrões de referência (HAIR et al., 2009): estatística qui-quadrado $\left(X^{2}\right)$, qui-quadrado/graus de liberdade $\left(X^{2} / g l<2\right)$, root mean square error of aproximation (RMSEA $\left.<0,100\right)$, root mean square residual (RMR < 0,050), normed fit index ( $\mathrm{NFI}>0,900)$, goodness off it índex (GFI >0,900), comparative fit index (CFI $>0,900)$, tucker lewis index (TLI >0,900), variância extraída $(>0,500)$, confiabilidade composta $(>0,700)$ e Alpha de Cronbach $(>0,700)$. 


\section{ANÁLISE E DISCUSSÃO DOS RESULTADOS}

\section{Caracterização geral dos respondentes}

Em relação à amostra investigada, foram entrevistados 242 estudantes de instituições de ensino localizadas em Santa Maria - RS, detentores de conta bancária. A Tabela 1 apresenta o perfil dos entrevistados, onde se pode constatar que a amostra foi composta por $34,7 \%$ de homens e $65,3 \%$ de mulheres.

Tabela 1 - Perfil dos entrevistados

\begin{tabular}{|c|c|c|c|}
\hline Variáveis & Entrevistados & Frequência & $\%$ \\
\hline \multirow{2}{*}{ Gênero (n = 242) } & Homens & 84 & 34,70 \\
\hline & Mulheres & 158 & 65,30 \\
\hline \multirow{4}{*}{ Idade $(n=223)$} & Até 20 anos & 56 & 25,10 \\
\hline & De 21 a 23anos & 67 & 30,00 \\
\hline & De 24 a 27 anos & 50 & 22,40 \\
\hline & Mais de 28 anos & 50 & 22,40 \\
\hline \multirow{3}{*}{$\begin{array}{l}\text { Estado Civil } \\
\qquad(n=238)\end{array}$} & Solteiro & 188 & 79,00 \\
\hline & Casado/União Estável & 46 & 19,30 \\
\hline & Outros & 4 & 1,70 \\
\hline \multirow{4}{*}{$\begin{array}{l}\text { Renda Familiar } \\
\qquad(n=179)\end{array}$} & Até $\mathrm{R} \$ 1400$ & 46 & 25,70 \\
\hline & de $R \$ 1401$ a 2700 & 44 & 24,60 \\
\hline & de $R \$ 2701$ a 4500 & 46 & 25,70 \\
\hline & Acima de $\mathrm{R} \$ 4501$ & 43 & 24,00 \\
\hline \multirow{3}{*}{$\begin{array}{l}\text { Escolaridade } \\
\qquad(n=236)\end{array}$} & Ensino Médio & 78 & 33,10 \\
\hline & Graduação & 149 & 63,10 \\
\hline & Pós-graduação & 9 & 3,80 \\
\hline \multirow{4}{*}{$\begin{array}{l}\text { Banco que mais utiliza } \\
\qquad(\mathrm{n}=227)\end{array}$} & Banco do Brasil & 100 & 44,10 \\
\hline & Caixa Econômica Federal & 53 & 23,30 \\
\hline & Banrisul & 34 & 15,00 \\
\hline & Outros (Santander, Sicredi, Itaú entrs outros) & 40 & 17,60 \\
\hline
\end{tabular}

Fonte: elaborada pelos autores.

A grande maioria dos pesquisados possui até 27 anos de idade $(77,5 \%)$ e é solteira $(79 \%)$, sendo que 19,3\% dizem ser casados ou estar em união estável. Em relação à renda familiar, $26 \%$ dos entrevistados não responderam a questão, enquanto que entre os que responderam, destaca-se que $50,3 \%$ tem renda familiar de até $R \$ 2.700,00$.

Em relação ao grau de escolaridade dos entrevistados, 33,1\% estão cursando ensino médio, $63,1 \%$ estão cursando graduação e 3,8\% em nível de pós-graduação. Já em relação ao estabelecimento bancário que mais utilizam, destaca-se o Banco do Brasil com $44,1 \%$ dos entrevistados, enquanto que a Caixa Econômica Federal aparece em segundo lugar com 23,3\% e o Banrisul com 15\%, enquanto que outros bancos representam 17,6\%. 


\section{Resultados relativos ao modelo de formação da RSC}

A fim de testar o modelo híbrido de formação da RSC, num primeiro momento foi testado o modelo de mensuração de cada um dos quatro construtos estudados, quais sejam: percepção da RSC; congruência empresa-RSC; atribuição motivacional e credibilidade corporativa.

Neste sentido, foi realizada a Análise Fatorial Confirmatória (AFC) para cada um dos construtos analisados, cujos resultados estão apresentados na Tabela 2, onde são apresentados os índices de ajustamento para o modelo inicial e também para o modelo final, após os ajustes realizados.

O construto "Percepção da RSC" era originalmente constituído por 22 variáveis, sendo que foram excluídas as variáveis 01 a 13, por não apresentarem coeficientes padronizados dentro limite do mínimo desejável $(0,500)$. Dessa forma, permaneceram no modelo final as variáveis 14 a 22 , com correlação entre os erros 17 e 19, 20 e 21, 20 e 22 e 21 e 22. Com essas modificações, os índices de ajuste para este construto resultaram em valores dentro dos parâmetros mínimos desejáveis, com exceção da variância extraída, 0,020 abaixo do ideal, mas compensados pelos bons índices da confiabilidade composta e alpha de Cronbach.

O construto "Congruência" era composto por 4 variáveis, sendo que a variável 3 não apresentou coeficiente padronizado em nível aceitável (maior do que 0,500), sendo retirada do estudo. Os índices apresentaram-se todos dentro dos padrões estabelecidos.

O construto "Atribuição Motivacional", também era composto por 4 variáveis, sendo excluída a variável 1 pois não apresentou coeficiente padronizado em nível aceitável (maior do que 0,500). Com isso, os índices apresentaram-se dentro dos padrões, com exceção da variância extraída que ficou abaixo de 0,500 e o alpha de Cronbach com valor de 0,650, considerado baixo para alguns autores, mas "aceitável desde que outros indicadores sejam bons" (HAIR et. al., 2009, p. 592). A confiabilidade composta obteve 0,700 , considerado bom, portanto, para fins deste estudo considerar-se-á que este construto possui consistência interna.

Já o construto "Credibilidade Corporativa", não houve necessidade de nenhuma exclusão de variáveis, sendo necessário apenas a correlação entre os erros 1 e 2. Quanto aos índices, $\chi^{2} / \mathrm{GL}$ resultou acima do recomendado, já os demais se apresentaram dentro dos padrões mínimos desejáveis.

Pode-se constatar que para a validação dos construtos estudados, foram necessárias algumas modificações nos mesmos para que fossem alcançados coeficientes de ajuste minimamente satisfatórios. Ao excluir variáveis e/ou inserir correlações, modifica-se a estrutura do construto proposta no modelo original. Especialmente no caso da exclusão de variáveis, pode-se estar perdendo parte da própria definição conceitual do construto, ao se retirar da análise a dimensão que a variável excluída contempla.

Nesse sentido, é importante considerar que, apesar de ter se obtido a validação de todos os construtos propostos no modelo de Pérez e Rodríguez del Bosque (2013), não foram validados os construtos na sua composição original. Esse fato reforça a necessidade de realização de estudos futuros para avaliar a aplicabilidade do modelo em outros contextos. 


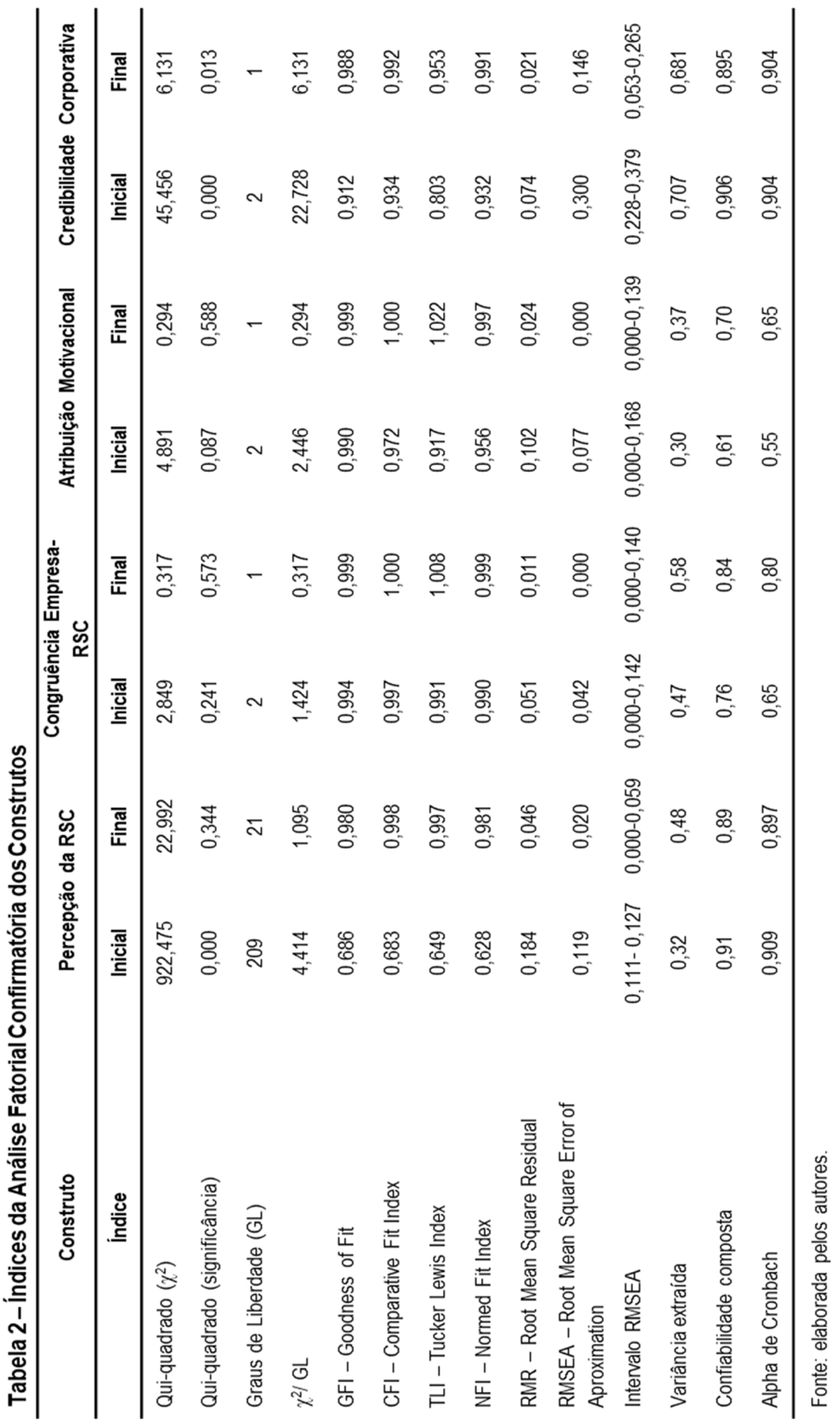


O modelo híbrido final está representado na Figura 1 e os índices na Tabela 3. Todos os índices apresentaram-se dentro dos padrões estabelecidos, mostrando a consistência do modelo.

Tabela 3 - Índices do modelo híbrido

\begin{tabular}{|c|c|c|}
\hline Índice & Inicial & Final \\
\hline Qui-quadrado $\left(\chi^{2}\right)$ & 302,644 & 259,584 \\
\hline Qui-quadrado (significância) & 0,000 & 0,000 \\
\hline Graus de Liberdade (GL) & 139 & 140 \\
\hline$\chi^{2} / \mathrm{GL}$ & 2,177 & 1,854 \\
\hline GFI - Goodness of Fit & 0,887 & 0,902 \\
\hline CFI - Comparative Fit Index & 0,938 & 0,955 \\
\hline TLI - Tucker Lewis Index & 0,924 & 0,945 \\
\hline $\mathrm{NFI}$ - Normed Fit Index & 0,892 & 0,908 \\
\hline RMR - Root Mean Square Residual & 0,134 & 0,109 \\
\hline RMSEA - Root Mean Square Error of Aproximation & 0,070 & 0,060 \\
\hline Intervalo RMSEA & $0,059-0,081$ & $0,048-0,071$ \\
\hline
\end{tabular}

Fonte: elaborada pelos autores.

Continuando com os procedimentos de análise de dados, partiu-se para a análise das hipóteses, ou seja, comprovar relações entre os construtos. Os resultados da análise confirmam as relações propostas entre as três primeiras construções: congruência empresa-RSC, atribuição motivacional e credibilidade da empresa, da mesma forma mostraram que a congruência empresa - RSC contribui diretamente para a geração de percepção positivas da RSC. A influência direta da atribuição motivacional na percepção da RSC não foi possível confirmar, sendo rejeitada a hipótese. A Figura 1 apresenta o modelo com os valores encontrados no estudo de Pérez e Rodríguez del Bosque (2013) e os resultados alcançados no presente estudo.

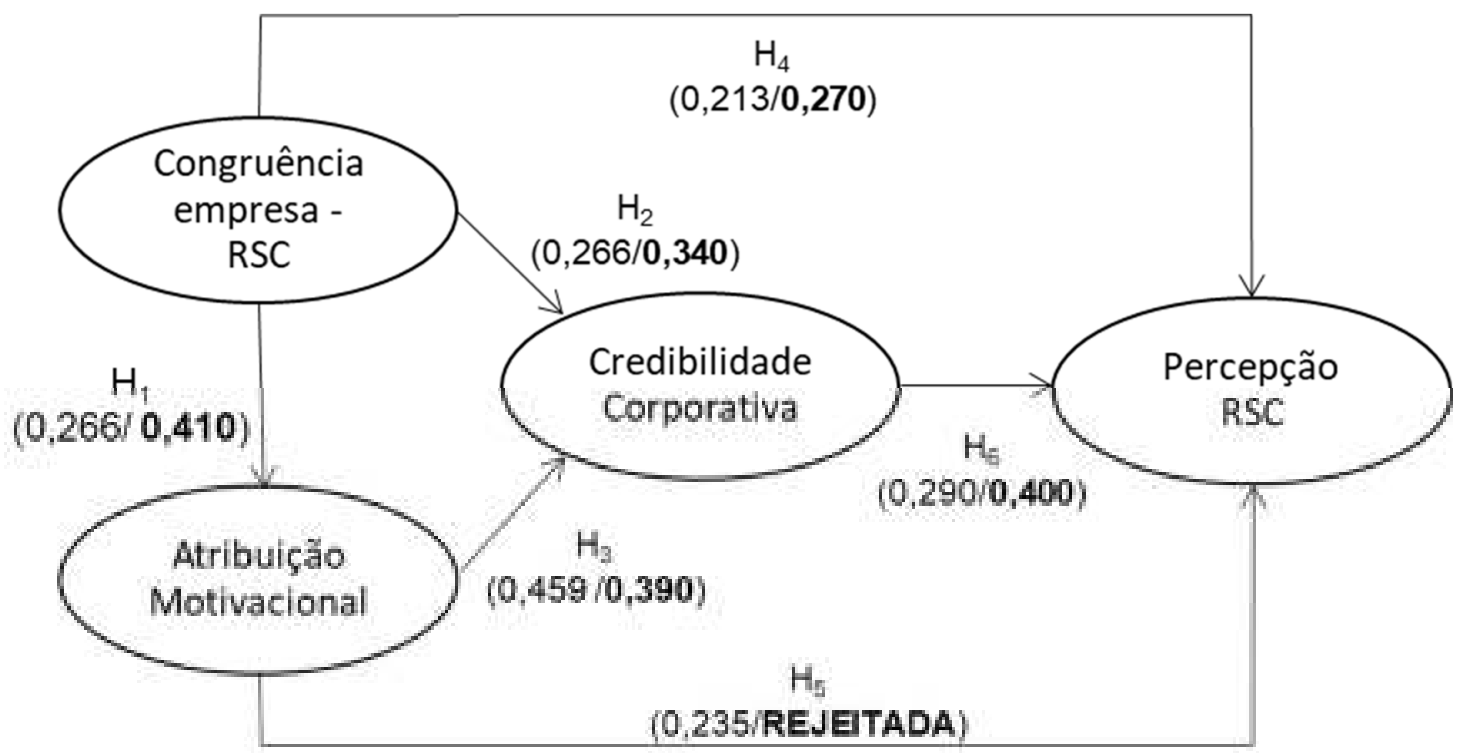

Figura 1 - Índices do modelo original e híbrido

Fonte: elaborada pelos autores. 
No estudo da correlação, Pestana e Gageiro (2000) apresentam parâmetros para analisar a força da relação entre duas variáveis, apontando que se for menor do que 0,200 , a associação pode ser considerada muito baixa; entre 0,200 e 0,390 é considerada baixa; entre 0,400 e 0,690 é moderada; 0,700 a 0,890 alta e acima de 0,900, muito alta.

Os resultados encontrados indicam que a congruência empresa- RSC influencia positivamente e de forma moderada a atribuição motivacional $(\beta=0,410 ; p<0,050)$, confirmando a Hipótese 1. Se comparado com os resultados encontrados no estudo de Pérez e Rodríguez del Bosque (2013) $(\beta=0,266 ; p<0,050)$, obteve-se no presente estudo uma influência mais forte, indicando um melhor resultado. A confirmação desta hipótese reforça o papel mediador das atribuições motivacionais do consumidor para os efeitos da congruência sobre a formação das percepções de RSC, conforme encontrado nos estudos de Rifon et al. (2004).

A segunda hipótese $\left(\mathrm{H}_{2}\right)$ também foi confirmada $(\beta=0,340 ; p<0,050)$, indicando que a congruência empresa-RSC exerce baixa influência na credibilidade corporativa. Da mesma forma que na hipótese anterior, o estudo de Pérez e Rodríguez del Bosque (2013) obteve um resultado inferior ( $\beta=0,266 ; p<0,050$ ), indicando uma força menor nesta relação. Esse resultado mostra que quanto mais o cliente perceber uma congruência nas ações da organização com a RSC, maior será a credibilidade percebida.

A influência da atribuição motivacional sobre a credibilidade corporativa (Hipótese 3 ) também foi confirmada $(\beta=0,390 ; p<0,050)$, apesar de indicar uma relação mais fraca do que a encontrada no estudo de Pérez e Rodríguez del Bosque (2013) $(\beta=0,459 ; p<0,050)$. Essa relação confirma que a percepção dos consumidores com relação as motivações das empresas em suas ações, em termos de serem mais altruístas ou não, por exemplo, influencia de forma direta sua percepção sobre a credibilidade da organização em suas ações de RSC.

Partindo-se para a análise dos antecedentes diretos da percepção da RSC, os resultados apontam que a congruência empresa-RSC influencia a percepção RSC $(\beta=0,270 ; p<0,050)$, confirmando a Hipótese 4 deste estudo. Ao confrontar com os resultados do estudo de Pérez e Rodríguez del Bosque (2013) $(\beta=0,213 ; p<0,050)$, observa-se um melhor resultado, visto que a força da relação no presente estudo, ainda que segundo Pestana e Gageiro (2000) seja considerada baixa, neste estudo foi maior. Conclui-se, portanto, que quanto mais alinhada estiver a instituição financeira com ações de responsabilidade social que sejam positivamente avaliados pelos consumidores, mais positiva será a percepção dos seus clientes com relação a estas ações.

A quinta hipótese $\left(\mathrm{H}_{5}\right)$ afirma que a atribuição motivacional irá influenciar a percepção RSC, o que não se confirma no presente estudo. O estudo de Pérez e Rodríguez del Bosque (2013) encontrou uma relação fraca. Dessa forma, neste estudo não foi possível confirmar que a percepção dos consumidores com relação às motivações da empresa exerça influência direta sobre sua percepção com relação a RSC. Portanto, neste estudo, a atribuição motivacional não se apresentou como um preditor da formação da imagem de RSC.

A última hipótese $\left(\mathrm{H}_{6}\right)$ também foi confirmada, indicando que a credibilidade corporativa influencia de forma moderada a percepção RSC $(\beta=0,400 ; p<0,050)$. Se comparado com o modelo original $(\beta=0,290$; $p<0,050$ ), obteve-se no presente estudo um melhor resultado. Comprova-se, assim, que quanto maior for a credibilidade da organização percebida por seus clientes, mais intensa será a percepção com relação a RSC. 


\section{CONSIDERAÇÕES FINAIS}

Os resultados deste estudo mostraram-se consistentes com os encontrados no estudo de Pérez e Rodríguez del Bosque (2013) utilizado como referência. Praticamente todas as hipóteses testadas foram confirmadas. Apenas não foi confirmada a influência da atribuição motivacional na percepção da RSC. Isso implica em que a forma como os clientes percebem as motivações dos bancos para suas ações sociais não influencia na percepção que possuem sobre a responsabilidade social dessas organizações. Ou seja, a RSC percebida pelos clientes bancários não apresenta dependência direta de seus julgamentos sobre as motivações dos bancos para estas ações socialmente responsáveis. Porém, confirmou-se uma dependência indireta, uma vez que estes julgamentos afetam a credibilidade dos bancos, que por sua vez afeta a percepção da RSC, conforme hipótese confirmada neste estudo.

Os resultados encontrados apresentam implicações significativas para as estratégias de gestão da RSC nas organizações em geral, especialmente nas instituições financeiras, objeto de análise neste estudo. Constatou-se que é importante que organizações promovam suas iniciativas de RSC especialmente concentradas em demonstrar a ausência de motivações auto-interessadas.

Os resultados deste estudo confirmam o valor que traz para a instituição bancária envolver-se com ações de RSC consistentes com suas atividades comerciais e com seus produtos/serviços, melhorando a percepção de motivos altruístas por parte da organização assim como sua credibilidade quando se envolve neste tipo de atividade.

Cabe ressaltar que, apesar de ter se obtido a validação de todos os construtos propostos no modelo de Pérez e Rodríguez del Bosque (2013), não foram validados os construtos na sua composição original. Esse fato também pode ter influenciado a confirmação ou não das hipóteses testadas, reforçando a necessidade de realização de estudos futuros para avaliar a aplicabilidade do modelo em outros contextos. 


\section{REFERÊNCIAS}

ASHLEY, P.; QUEIROZ, A.; CARDOSO, A.; SOUZA, A.; TEODÓSIO, A.; BORINELLE, B.; VENTURA, E.; CHAVES, J.; VELOSO, L.; ALIGLERI, L.; LIMA, P.; FERREIRA, R. Ética e responsabilidade social nos negócios. São Paulo: Saraiva, 2003.

BIGNÉ, E.; CURRÁS, R.; SÁNCHEZ, I. Brand credibility in cause-related marketing: the moderating role of consumer values. Journal of Product and Brand Management, v.18, n. 6, p. 437-447, 2009.

BONN, I.; FISHER, J. Sustainability: the missing ingredient in strategy. Journal of Business Strategy, v. 32, n. 1, p. 5-14, 2011.

BRAVO, R.; MATUTE, J.; PINA, J. M. Corporate social responsibility as a vehicle to reveal the corporate identity: a study focused on the websites of Spanish Financial Entities. Journal of Business Ethics, v. 107, n. 2, p. 129-146, 2012.

CARROLL, A. B. A three-dimensional conceptual model of corporate social performance. Academy of Management Review, v. 4, p. 497-505, 1979.

CARROLL, A. B. Corporate Social Responsibility: evolution of a definitional construct. Business and Society, v. 38, n. 3, p. 268-295, 1999.

CRISÓSTOMO, V. L.; FREIRE, F. D. S.; VASCONCELLOS, F. C. Corporate social responsibility, firm value and financial performance in Brazil. Social Responsibility Journal, v. 7, n. 2, p. 295-309, 2011.

CRISÓSTOMO, V. L.; FREIRE, F. D. S.; SOARES, P. M. Uma Análise Comparativa da Responsabilidade Social Corporativa entre o Setor Bancário e outros no Brasil. Revista Contabilidade Vista \& Revista, v. 23, n. 1, p. 103-128, out./dez. 2012.

CURRÁS, R. Identidad e imagen corporativas: revision conceptual e interrelación. Teoría y Praxis, v. 7, p. 9-34, 2010.

DAHLSRUD, A. How corporate social responsibility is defined: an analysis of 37 definitions. Corporate Social Responsibility and Environmental Management, v. 15, n. 1, p. 1-13, 2008.

DELIOS, A. How can organizations be competitive but dare to care? Academy of Management Perspectives, v. 24, n. 3, p. 25-36, 2010.

DYLLICK, T.; HOCKERTS, K. Beyond the business case for corporate sustainability. Business Strategy and the Environment, v. 11, n. 2, p. 130-141, 2002.

DUNPHY, D. Corporate sustainability: challenge to managerial orthodoxies. Journal of the Australian and New Zeland Academy of Management, v. 9, n. 1, p. 2-11, 2003.

GUIMARÃES FILHO, A. D. J.; GOMES, E. R. Impactos internos das várias formas organizacionais de atuação em responsabilidade social corporativa nos grandes bancos no Brasil. Revista ADMpg Gestão Estratégica, v. 3, n. 1, p.7381, 2010.

HAIR, F. et al. Análise multivariada de dados. 6 ed. Porto Alegre: Bookman, 2009.

HART, S. L.; MILSTEN, M. B. Creating sustainable value. Academy of Management Executive, v. 17, n. 2, Info, p. 56-67, 2010.

HE, H.; BALMER, J. M. T. Identity studies: multiple perspectives and implications for corporate-level marketing. European Journal of Marketing, v. 41, n. 7/8, p. 765-785, 2007.

HEIDER, F. The psychology of interpersonal relations. Hillsdale, NJ: Lawrence Erlbaum Associates, 1958.

HEDSTRÖM, G.; POLTORZYCKI, S.; STROH, P. Sustainable development: the next generation. Prism - Sustainable Development: How Real, how Soon, and Who's Doing What? n. 4, p. 5-19, 1998.

IDOWU, S. O.; PAPASOLOMOU, I. Are the corporate social responsibility matters based on good intentions or false pretences? An empirical study of the motivations behind the issuing of CSR reports by UK companies. Corporate Governance, v. 7, n.2, p. 136-147, 2007.

KELLEY, H. H. Processes of causal attribution. American Psychologist, v. 2, n. 28, p.107-128, 1973.

LEMME, C. F. Sustentabilidade e Finanças. In: GARDETTI, M. A. (Org.) Textos en sustentabilidad empresarial: integrando las consideraciones sociales, ambientales y económicas com el corto y largo plazo. Buenos Aires: La-Bell, p. 129-169, 2005.

LYRA, F. R.; SOUZA, M. J. B. Percepção das práticas de responsabilidade social corporativa pelos stakeholders do Parque Beto Carrero World. In: ENANPAD, 37, 2013, Rio de Janeiro. Anais... Rio de Janeiro, 2013.

MARCO, G. J., HOLLINGWORTH, R. M., DURHAM, W. Silent spring revisited. Washington, DC: American Chemical Society, 1987.

MARCZYK, G., DEMATTEO, D., FESTINGER, D. Essentials of research design and methodology. John Wiley \& Sons: Hoboken, New Jersey, 2005. 
MATOS, F. G. Ética empresarial e responsabilidade social. Revista Recre@rte, n.3, jun.2005.

MORIMOTO, R; ASH, J.; HOPE, C. Corporate social responsibility audit: from theory to practice. Journal of Business Ethics, v. 62, n. 4, p. 315-325, 2005.

NAN, X.; HEO, K. Consumer responses to corporate social responsibility (CSR) initiatives: examining the role of brandcause fit in cause-related marketing. Journal of Advertising, v. 36, n. 2, p. 63-74, 2007.

ÖBERSEDER, M.; SCHLEGELMILCH, B. B.; MURPHY, P. E. CSR practices and consumer perceptions. Journal of Business Research, v. 66, n. 10, p. 1839-1851, 2013.

PARK, J.; LEE, H.; KIM, C. Corporate social responsibilities consumer trust and corporate reputation: South Korean consumers' perspectives. Journal of Business Research, v. 67, n. 3, p. 295-302, 2014.

PEDERSEN, E. R.; NEERGAARD, P. What matters to managers? The whats, whys, and hows of corporate social responsibility in a multinational corporation. Management Decision, v. 47, n. 8, p. 1261-1280, 2009.

PÉREZ, A.; RODRÍGUEZ DEL BOSQUE, I. Customer personal features as determinants of the formation process of corporate social responsibility perceptions. Psychology and Marketing, v. 30, n 10, p. 903-917, 2013.

PESTANA, M. H., GAGEIRO, J. N. Análise de dados para as Ciências Sociais: a complementariedade do SPSS. Lisboa: Sílabo, 2000.

RIFON, N. J.; CHOI, S. M.; TRIMBLE, C. S.; LI, H. Congruence effects in sponsorship: the mediating role of sponsor credibility and consumer attributions of sponsor motive. Journal of Advertising, n.33, p.29-42, 2004.

SANDHU, S. Shifting paradigms in corporate environmentalism: from poachers to gamekeepers. Business and Society Review, v. 115, n. 3, p. 285-310, 2010.

SEN, S.; BHATTACHARYA, C. B. Does doing good always lead to doing better? Consumer reactions to corporate social responsibility. Journal of Marketing Research, v. 38, n. 2, p. 225-43, 2001.

SERPA, D. A. F; FOURNEAU, L. F. Responsabilidade social corporativa: uma investigação sobre a percepção do consumidor. Revista de Administração Contemporânea, v. 11, n. 3, p. 83-103, Jul./Set., 2007.

VAN MARREWIJK, M. Concepts and definitions of CSR and corporate sustainability: Between agency and communion. Journal of Business Ethics, v. 44, n. 2, p. 95 - 105, 2003.

WCED - United Nations. Report of the World Commission on Environment and Development, General Assembly Resolution, 42/187, 11, Retrieved: 2077, p. 11-14, December 1987. 